\title{
Oldhamianoside inhibits the growth of ovarian cancer both in vitro and in vivo via adjusting inflammation and angiogenesis signals
}

This article was published in the following Dove Press journal:

OncoTargets and Therapy

\author{
Zenghui $\mathrm{Li}^{1, *}$ \\ Ying Zuol,* \\ Li Hou' \\ Liangliang Dong ${ }^{2}$ \\ Xiaomei Sun ${ }^{3}$ \\ 'Department of Gynecology, \\ The Affiliated Yantai Yuhuangding \\ Hospital of Qingdao University, \\ Yantai, 264000, China; '2Department \\ of Medical Oncology, The Affiliated \\ Yantai Yuhuangding Hospital of \\ Qingdao University, Yantai, 264000, \\ China; ${ }^{3}$ Department of Surgery, \\ The Affiliated Yantai Yuhuangding \\ Hospital of Qingdao University, Yantai, \\ 264000, China \\ *These authors contributed equally \\ to this work
}

Objective: The aim of this study was to determine the effects and possible mechanisms of oldhamianoside on the growth of human ovarian cancer both in vitro and in vivo.

Materials and methods: CCK- 8 assay was applied to estimate the effect of oldhamianoside on cell proliferation inhibition in vitro. Nude mice bearing human ovarian SKOV3 xenograft tumors were treated with oldhamianoside to investigate the effects of compound administration on tumor growth in vivo. To further investigate the mechanisms of inhibition effects of oldhamianoside on ovarian cancer growth in vivo, the levels of TNF- $\alpha$, IL-6, and MCP-1 in plasma from the mice were measured by ELISA. Western blot was used to detect the expression of angiogenesis- and/or apoptosis-related proteins.

Results: We found that oldhamianoside treatment inhibited SKOV3 proliferation and growth both in vitro and in vivo. Meanwhile, the levels of TNF- $\alpha$, IL-6, and MCP-1 in plasma were markedly suppressed in oldhamianoside-treated mice. Additionally, oldhamianoside treatment inhibited the expression of VEGF and VEGFR2 and decreased the expression of caspase-3 and $\mathrm{Bax} / \mathrm{Bcl}-2$ ratio.

Conclusion: Our data indicate that oldhamianoside has an obvious inhibition effect on SKOV3 proliferation, and the mechanisms might be related to inhibition of cell growth, apoptosis induction, and adjusting the inflammatory response and angiogenesis signal.

Keywords: oldhamianoside, ovarian cancer, SKOV3, inflammation, VEGF, VEGFR2

\section{Introduction}

Ovarian cancer remains one of the main causes of gynecological cancer deaths in women worldwide. ${ }^{1}$ It is estimated that the 5-year survival rate for ovarian cancer is $25 \%$ for advanced-stage disease, which is when $85 \%$ of the patients are diagnosed. ${ }^{2}$ The poor survival rate is primarily due to our poor understanding of the disease in addition to the asymptomatic nature of this cancer in the early stages. In recent years, cytoreductive surgery and chemotherapy with platinum are the recommended standard treatments for advanced ovarian cancer. ${ }^{3}$ Unfortunately, more than half of the advanced ovarian cancer patients will ultimately develop recurrence and acquire a chemoresistance to platinum. ${ }^{4,5}$ The treatment of these platinum-refractory patients therefore requires the development of alternative therapies.

Gypsophila oldhamiana, a plant which is rich in saponin, has been used in the treatment of fever, consumptive diseases, and infantile malnutrition in Chinese. ${ }^{6}$ Previous phytochemical studies on this species had reported that a new triterpenoid saponin called oldhamianoside possessed many pharmacological activities, including antioxidant, immunomodulatory, and antitumor effects..$^{5,7}$ It is well known that
Department of Surgery, The Affiliated

Yantai Yuhuangding Hospital of Qingdao University, No 20 Yuhuangding

East Road, Zhifu District, Yantai, 264000, China

Tel +86 I 5853529296

Email sunxiaomei2233@163.com 
saponin could exert antitumor activity by several mechanisms, which include cell cycle arrest, growth factor signaling modulation, cellular migration, angiogenesis, and apoptosis induction. Some studies also indicated that oldhamianoside can effectively inhibit tumor growth, induce tumor cell apoptosis, prevent tumor angiogenesis, and inhibit cancer cell migration. ${ }^{7,8}$ In this study, we explore the growth inhibition effect of oldhamianoside in human ovarian cancer SKOV3 cells and the nude mice xenograft model and further examine the molecular mechanisms of oldhamianoside.

\section{Materials and methods Chemicals}

Oldhamianoside was isolated from G. oldhamiana, and the purity of oldhamianoside as measured by high-performance liquid chromatography was $>98 \%$.

\section{Cell culture}

Human ovarian cancer SKOV3 cells were obtained from Wuhan Boster Biological Engineering Co., Ltd (Wuhan, Hubei, China). Cells were routinely cultured in RPMI-1640 medium supplemented with $10 \% \mathrm{FBS}, 100 \mathrm{U} / \mathrm{mL}$ penicillin, and $100 \mu \mathrm{g} / \mathrm{mL}$ streptomycin, at a $37^{\circ} \mathrm{C}$ humid atmosphere with $5 \% \mathrm{CO}_{2}$ and $95 \%$ air.

\section{CCK-8 assay}

Cells were seeded in 96 -well plates $\left(5 \times 10^{3}\right.$ per well) and incubated with increasing concentrations of oldhamianoside $(5,10,20,40,80$, and $160 \mu \mathrm{M})$ for 24,48 , and 72 hours, respectively. The controls were treated with an equal volume of compound vehicle DMSO. Cell viability was evaluated by a WST-8 Cell Counting Kit-8 (Dojindo, Kumamoto, Japan). Briefly, CCK-8 solution ( $10 \mu \mathrm{L})$ was added to each well after the corresponding compound and the cultures were incubated at $37^{\circ} \mathrm{C}$ for 1 hour. Absorbance at $450 \mathrm{~nm}$ was measured using a THERMOmax microplate reader (Molecular Devices LLC, Sunnyvale, CA, USA).

\section{Establishment of a xenograft mice model and grouping}

BALB/C nude mice (female, 4-6 weeks old, 16-17 g body weight) were purchased from Changzhou Card Vince Laboratory Animal Co., Ltd (SPF grade, Certificate No SCXK20150004; Changzhou, China). All animals were housed under pathogen-free conditions with a 12-hour light/dark cycle. All animal procedures were performed and approved by the institutional guidelines of Animal Care and Use Committee at Shandong Academy of Medical Science.
To establish an SKOV3 tumor implantation mouse model, SKOV3 cell suspension $\left(1 \times 10^{6}\right.$ cells per $0.2 \mathrm{~mL}$ per mouse $)$ was injected subcutaneously in the scapular region. Mice were then randomized into the following five groups ( $n=12$ each group): model group; cis-diamminedichloroplatinum II dichloride (DDP) group (positive control for the tumor inhibition efficiency); $5.0 \mathrm{mg} / \mathrm{kg}$ oldhamianoside group; $10.0 \mathrm{mg} / \mathrm{kg}$ oldhamianoside group; and $20.0 \mathrm{mg} / \mathrm{kg}$ oldhamianoside group. The dose of oldhamianoside was chosen based on our primary experiments. One week after the implantation, mice in the oldhamianoside group were intraperitoneally injected oldhamianoside $(5.0,10.0$, and $20.0 \mathrm{mg} / \mathrm{kg}$ ) for 10 days. Mice in the DDP group were administered DDP at a dose of $1.5 \mathrm{mg} / \mathrm{kg}$ for 10 days. Solvent alone was given in the model group.

The sizes of tumors and body weights were measured prior to treatment and twice a week once the treatment was initiated. The volume was measured by a caliper and calculated using the formula $\mathrm{V}=(\mathrm{ab} 2) / 2$, where " $\mathrm{a}$ " is the long axis and " $b$ " is the short axis. Mice were checked daily for any signs of illness. After administration of compound for 10 days, mice were euthanized via cervical dislocation, and the tumors were removed for weighing and further analysis. The inhibition rate of tumor growth was defined as a percentage of model control tumor weight. Blood samples were collected for biochemical analysis.

\section{Histological evaluation}

Tumor tissues were fixed in neutral formalin. Four $\mu \mathrm{m}$ thick sections were prepared and stained with HE solution. Pathological changes were observed under a light microscope.

\section{Quantification of inflammatory makers in animal models}

The level of TNF- $\alpha$ was measured using ELISA kit (Beijing BLKW Biotechnology Co., Ltd, Beijing, China) according to manufacturer's protocol. Levels of IL-6 and MCP-1 in plasma were estimated by ELISA according to the manufacturer's instructions (Nanjing Jiancheng Bioengineering Institute, Nanjing, China).

\section{Western blotting analysis}

Tumor tissues of the xenograft mice model from different groups were homogenized in RIPA lysis buffer, containing protease inhibitor cocktail EDTA-free, and then were centrifuged at 15,000 rpm for 15 minutes. The supernatants were collected, and the total protein concentration was determined 
using the bicinchoninic acid (BCA) kit (Chengdu Must Biotechnology Co., Ltd, Chengdu, China). Tumor lysates $(20 \mu \mathrm{g})$ were subsequently subjected to $12 \%$ SDS-PAGE gels and transferred to a nitrocellulose membrane. After blocking with $5 \%$ non-fat dry milk at room temperature for 2 hours, the primary antibodies of vascular endothelial growth factor (VEGF, 1:2,000; Cell Signaling Technology, Danvers, MA, USA), anti-phospho-VEGF receptor 2 (Cell Signaling Technology), caspase-3 (1:1,000; Cell Signaling Technology), Bcl-2 (1:1,000; Cell Signaling Technology), Bax (1:1,000; Cell Signaling Technology), and $\beta$-actin (1:1,000; Cell Signaling Technology) were added and incubated overnight. Subsequently, the membrane was then washed in TBS+Tween and incubated with horseradish peroxidase-conjugated secondary antibodies. Protein bands were detected by enhanced chemiluminescent reagents (Amersham Biosciences, Sydney, NSW, Australia).

\section{Statistical analysis}

Statistical analysis was implemented using SPSS 18.0 (SPSS Inc., Chicago, IL, USA) for windows. All data were reported as mean $\pm \mathrm{SD}$, and differences between groups were evaluated by one-way ANOVA followed by Tukey's multiple range test. A $P$-value of $<0.05$ was considered statistically significant.

\section{Results}

\section{Detection of retinoblastoma cell growth}

As shown in Figure 1, oldhamianoside inhibited SKOV3 cell proliferation in a dose- and time-dependent pattern. The inhibition rate was only $1.45 \%$ at the concentration of $5 \mu \mathrm{M}$

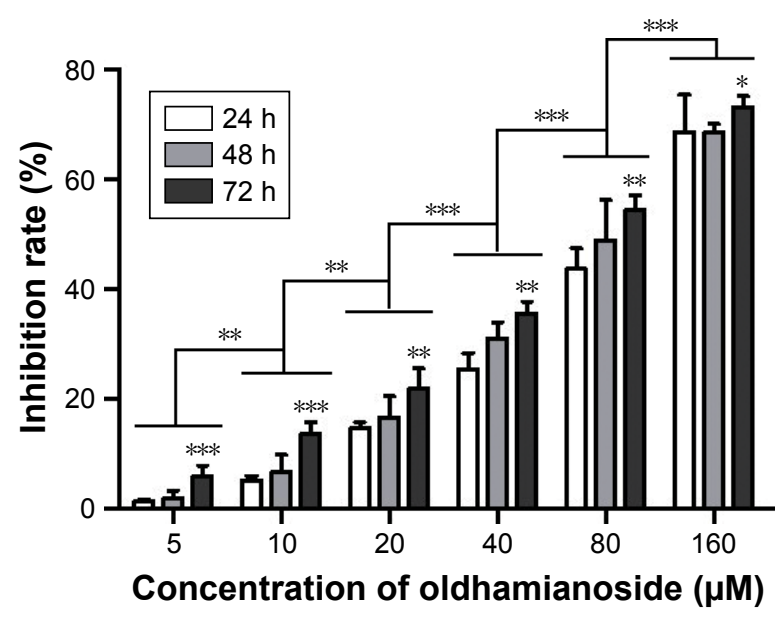

Figure I Oldhamianoside induced growth inhibition in SKOV3 cells. Notes: Cells were treated with increasing concentrations of oldhamianoside for up to 72 hours. Viable cells are reported as a percentage of untreated control. Bars indicate mean $\pm S D(n=3)$. $* P<0.05, * * P<0.01$, and $* * * P<0.001$ vs the model group. Abbreviation: h, hours. after 24 hours of treatment with oldhamianoside. Then, at a concentration of $160 \mu \mathrm{M}$, the inhibition rates were increased to $68.76 \%$ after 24 hours of treatment with oldhamianoside, $68.66 \%$ after 48 hours of treatment with oldhamianoside, and $73.27 \%$ after 72 hours of treatment with oldhamianoside, respectively (Figure 1). The results showed a notable inhibition effect of oldhamianoside on SKOV3 proliferation.

\section{Inhibitory effect of oldhamianoside on SKOV3 tumors in nude mice}

The inhibitory effect of oldhamianoside on SKOV3 tumors in nude mice was evaluated by the weight and volume of the tumor tissues. Two weeks after injecting SKOV3 cells, the weight of tumors in the model group increased to $1.84 \pm 0.31 \mathrm{~g}$, whereas $5.0,10.0$, and $20.0 \mathrm{mg} / \mathrm{kg}$ oldhamianoside significantly reduced the tumor weight to $0.96 \pm 0.18 \mathrm{~g}$ (47.83\% inhibition), $0.88 \pm 0.22 \mathrm{~g}(52.17 \%$ inhibition), and $0.78 \pm 0.21 \mathrm{~g}$ (57.61\% inhibition), respectively. Moreover, oldhamianoside treatment at different doses was generally well tolerated by mice with no apparent loss of body weight compared with the model group. DDP not only inhibited SKOV3 tumor growth with $60.87 \%$ inhibition but also significantly reduced the body weight of mice. The tumor volume was also assessed after a 2 -week growth. As shown in Table 1, the average volume of tumors in model mice was $898 \mathrm{~mm}^{3}$ after a 2-week growth, which was significantly reduced by oldhamianoside treatment.

To assess the effects of oldhamianoside on histological changes in SKOV3 tumors, HE staining was used. SKOV3 tumors from the model group showed significant inflammatory infiltration, diffuse distribution cells, round or oval cells with lumpy or nested arrangement, large nuclei stained and irregular, and nuclear cytoplasm ratio imbalance with tumor giant nuclear mitotic, whereas oldhamianoside treatment ameliorated cell morphology, and it showed marked upturn with gradual increases in the doses of oldhamianoside (Figure 2).

\section{Oldhamianoside decreased the inflammatory factors}

To identify the anti-inflammatory property of oldhamianoside, the levels of inflammatory mediators including TNF- $\alpha$, MCP-1, and IL-6 in plasma were measured by ELISA. As demonstrated in Figure 3, in contrast to the model group, the levels of above-mentioned factors were markedly decreased after being treated with oldhamianoside in a dose-dependent manner. 
Table I Inhibitory effects of oldhamianoside on SKOV3 tumors in nude mice (mean \pm SD)

\begin{tabular}{|c|c|c|c|c|}
\hline Groups & $\begin{array}{l}\text { Body weight }(\mathrm{g}) \\
\text { Initial/2 weeks }\end{array}$ & $\begin{array}{l}\text { Tumor } \\
\text { weight (g) }\end{array}$ & $\begin{array}{l}\text { Inhibition } \\
\text { rate (\%) }\end{array}$ & $\begin{array}{l}\text { Tumor } \\
\text { volume }\left(\mathrm{mm}^{3}\right)\end{array}$ \\
\hline Model group & $16.8 \pm 1.8 / 19.3 \pm 2.2$ & $|.84 \pm 0.3|$ & $\begin{array}{l}\text { This value } \\
\text { does not exist }\end{array}$ & $732.8 \pm 76.7$ \\
\hline DDP group & $16.9 \pm 1.6 / 16.6 \pm 2.2$ & $0.72 \pm 0.12$ & 60.87 & $276.3 \pm 43.2 * *$ \\
\hline \multicolumn{5}{|c|}{ Oldhamianoside } \\
\hline $5.0 \mathrm{mg} / \mathrm{kg}$ & $\mid 7.1 \pm 1.9 / 18.7 \pm 2.3$ & $0.96 \pm 0.18$ & $47.83^{\#}$ & $496.3 \pm 51.4 * *, \#$ \\
\hline $10.0 \mathrm{mg} / \mathrm{kg}$ & $16.8 \pm 2.3 / \mid 8.7 \pm 3.1$ & $0.88 \pm 0.22$ & 52.17 & $4 \mid 2.9 \pm 56.8 * *$ \\
\hline $20.0 \mathrm{mg} / \mathrm{kg}$ & $16.8 \pm 1.9 / 18.1 \pm 2.4$ & $0.78 \pm 0.21$ & 57.61 & $289.2 \pm 23.4^{* *}$ \\
\hline
\end{tabular}

Notes: Data are presented as mean \pm SD. ${ }^{* *} P<0.01$ vs model group; ${ }^{*} P<0.05$ vs the DDP group.

Abbreviation: DDP, cis-diamminedichloroplatinum II dichloride.

\section{Effects of oldhamianoside on VEGF and VEGFR2 expression in vitro and in ovarian tumor tissue}

The expression of VEGF in SKOV3 and in ovarian SKOV3 tumors was examined by Western blot. As shown in Figure 4, the expressions of VEGF and VEGFR were significantly decreased in ovarian tumorigenesis by oldhamianoside treatment in a dose-dependent manner.

\section{Effects of oldhamianoside on cell apoptosis}

Caspase- 3 is a critical executioner of apoptosis either partially or totally responsible for the proteolytic cleavage of many key proteins. The expression of caspase-3 was also detected, and our results revealed that the expression of both caspase- 3 and cleaved caspase-3 was elevated (Figure 5).

We also examined the protein levels of Bax and Bcl-2, which are known as the indicators of apoptosis. As shown in Figure 5, oldhamianoside treatment showed a significant increase in Bax and a significant decrease in Bcl-2 than in the model group. The $\mathrm{Bcl}-2 / \mathrm{Bax}$ ratio also decreased from 2.23 to 0.51 .

\section{Discussion}

In this study, we examined the inhibitory effects of oldhamianoside on tumor growth, inflammatory makers, angiogenesis, and apoptosis using an SKOV3 tumor mouse model. The results indicated that oldhamianoside delayed tumor growth and decreased the levels of MCP-1 and IL-6. Furthermore, oldhamianoside reduced the expression of VEGF in tumor tissues and decreased the $\mathrm{Bcl}-2 / \mathrm{Bax}$ ratio.

In an SKOV3 tumor xenograft model, oldhamianoside (5.0, 10.0, and $20.0 \mathrm{mg} / \mathrm{kg}$ ) suppressed ovarian tumor growth. Although the tumor growth inhibitory effect in the oldhamianoside group was less than that of the DDP group, it showed little effect on the loss of body weight. The tumor microenvironment, consisting of cancer and its surrounding
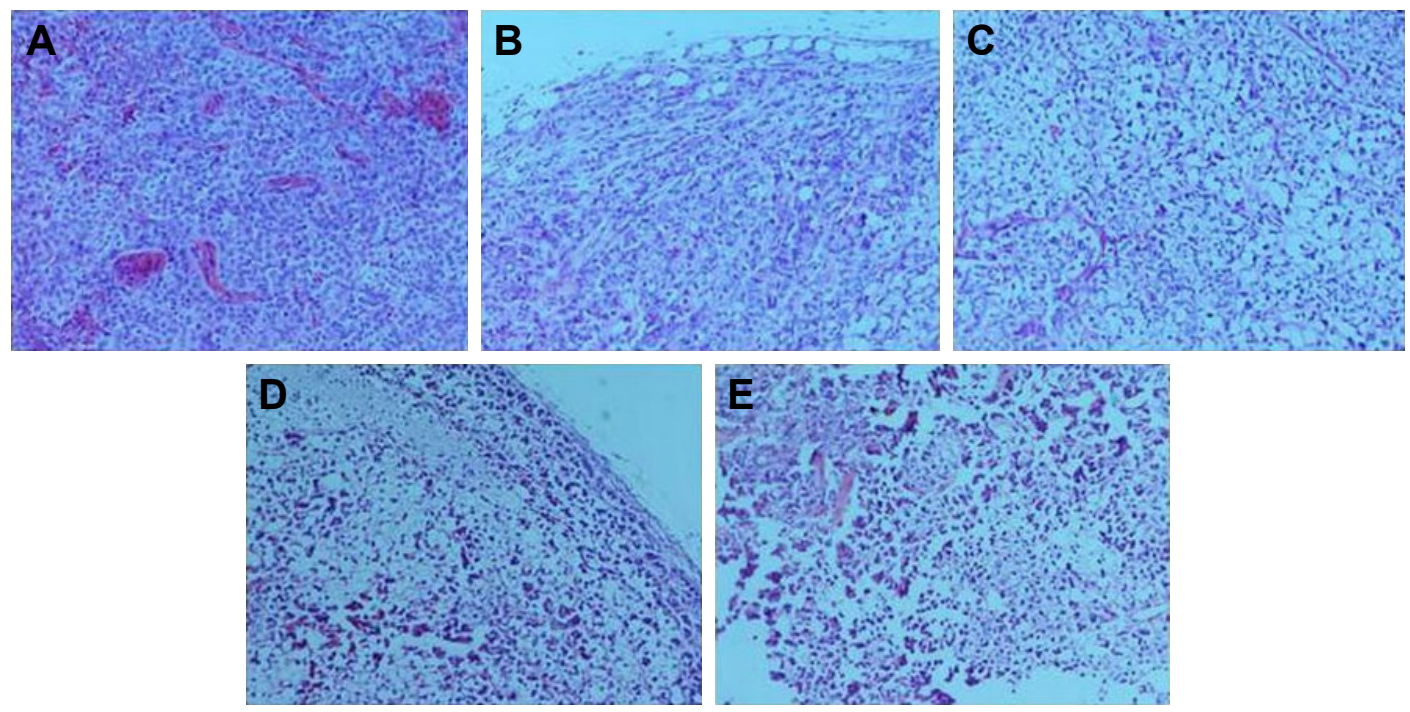

Figure 2 Effects of oldhamianoside on histopathological changes in SKOV3 tumors $(\times 200)$.

Notes: (A) Model group; (B) DDP group; (C) $5.0 \mathrm{mg} / \mathrm{kg}$ oldhamianoside group; (D) $10.0 \mathrm{mg} / \mathrm{kg}$ oldhamianoside group; and (E) $20.0 \mathrm{mg} / \mathrm{kg}$ oldhamianoside group. Abbreviation: DDP, cis-diamminedichloroplatinum II dichloride. 

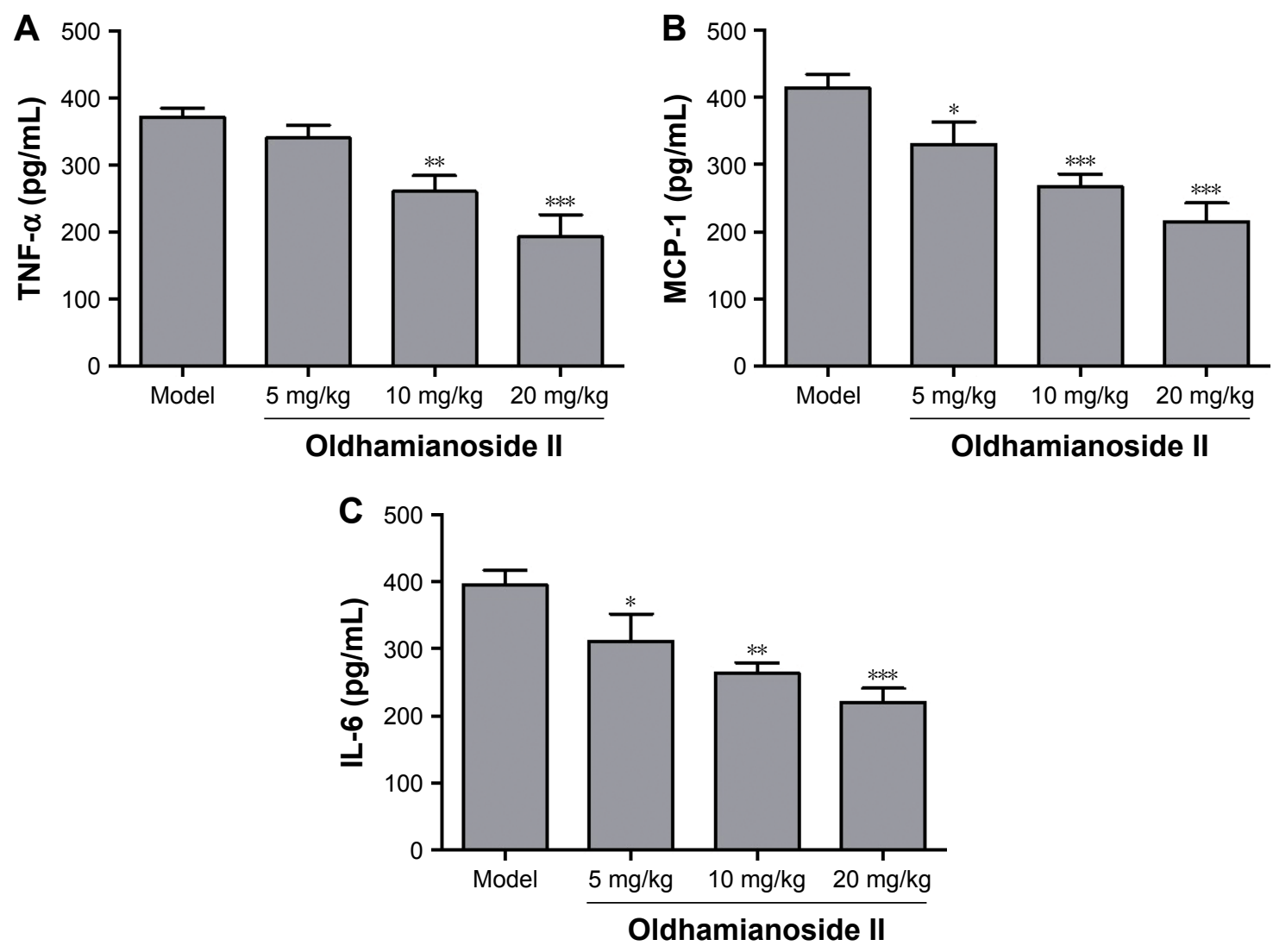

Figure 3 Oldhamianoside decreased (A) TNF- $\alpha$, (B) MCP-I, and (C) IL-6 levels in plasma.

Notes: Data are presented as mean \pm SD. $* P<0.05$, $* * P<0.01$, and $* * * P<0.001$ vs the model group. Experiments were performed in triplicate separately.

cells, soluble factors, and extracellular matrix, is increasingly recognized as a significant factor in cancer treatment response. ${ }^{9,10}$ Indeed, several anticancer agents have been demonstrated to exert anticancer activities by regulating the tumor microenvironment. ${ }^{11,12}$ Increase in inflammatory stress has been reported to be correlated with the development of different types of cancers, and several studies suggested that chronic inflammation plays an important role in cancer development. TNF has been shown to induce VEGF and other cytokines. In this regard, we speculate that oldhamianoside might affect not only tumor cells but also other cells, such as macrophages, in animal models. Oldhamianoside might also have multiple effects in ovarian cancer cells; in addition to direct growth inhibition, it might affect the growth and inflammatory factors produced by cancer cells. IL- 6 and MCP-1 are two important chemokines, which play critical roles in regulating host immune responses. Research has shown that MCP-1 and IL-6 may greatly participate in the development of different cancers. ${ }^{13,14}$ Our results showed that TNF- $\alpha$, MCP-1, and IL- 6 were reduced by oldhamianoside, which suggested that the inhibitory effect of ovarian cancer might be associated with the decrease in inflammation cytokine production.
Angiogenesis, a key step in tumor growth, can be induced by pro-angiogenic factors such as the fibroblast growth factor, angiopoietin, platelet-derived growth factor, and VEGF. VEGF has been shown to support both physiological vasculogenesis and cancer vascular network and is upregulated in many cancer cell types. ${ }^{15}$ Yamamoto et $\mathrm{al}^{16}$ found that VEGF contributes to tumor progression in a vast majority of ovarian tumors. The stimulation of endothelial cell motility and the growth of newly formed vessel formation are mediated by its activation of $m$-calpain via protein kinase A (PKA) activation. ${ }^{17}$ The VEGF family members induce proliferation, migration, and differentiation of endothelial cells, the major component of angiogenesis and lymphogenesis, by binding to VEGF receptor tyrosine kinases on endothelial cells. ${ }^{18,19}$ As knowledge on the multistep process of angiogenesis accumulates, more is being understood about how other pathways within the cell that have to be dysregulated in cancer, such as apoptosis, interact with angiogenesis. ${ }^{20}$ Our results confirmed that oldhamianoside treatment clearly reduced the expression of VEGF, which allowed us to elucidate a novel antitumorigenic and anti-angiogenic feature of oldhamianoside. 

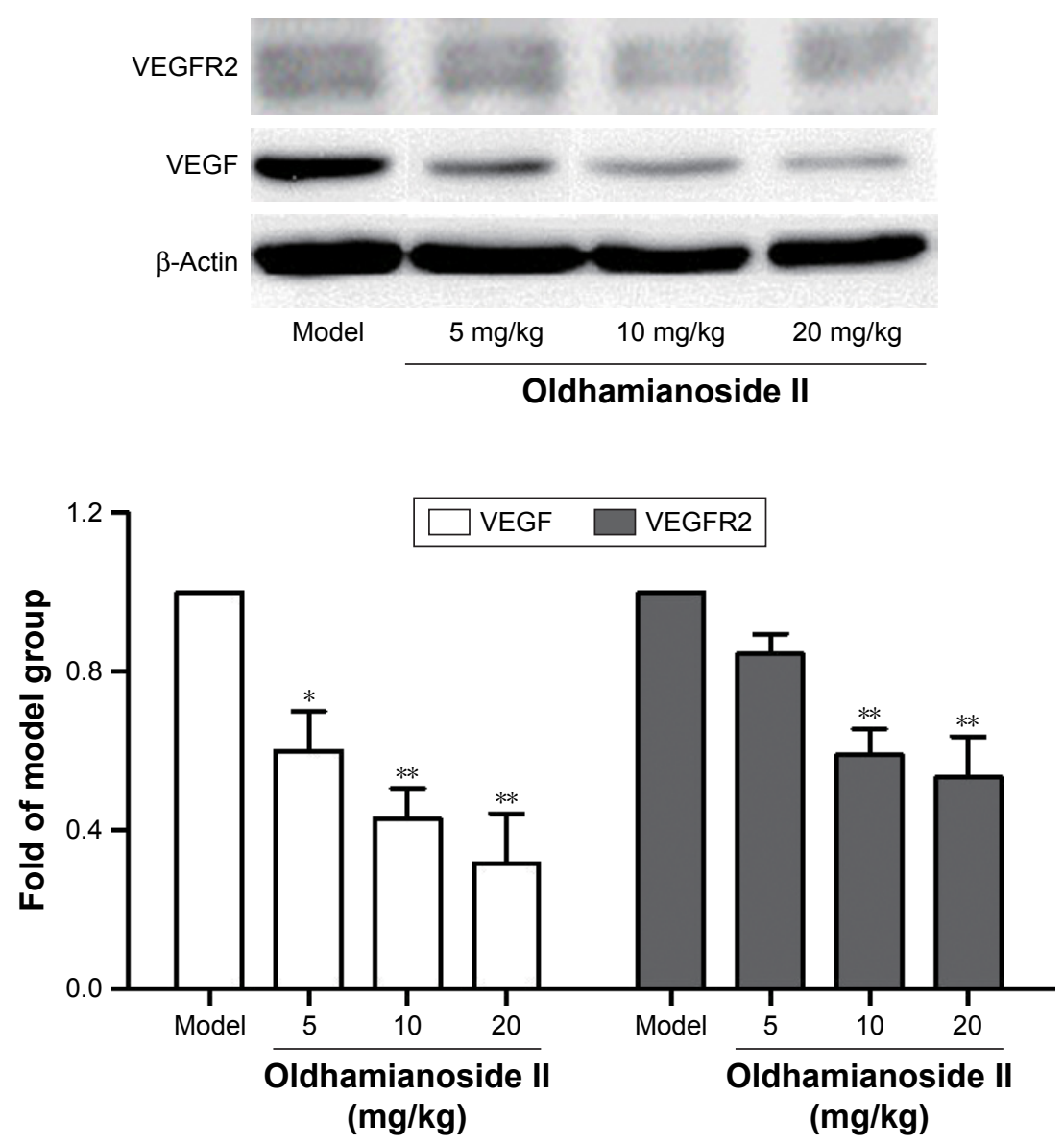

Figure 4 Oldhamianoside decreased VEGF and VEGFR2 expression.

Notes: Data are presented as mean \pm SD. $* P<0.05$ and $* * P<0.01$ vs the model group.

A
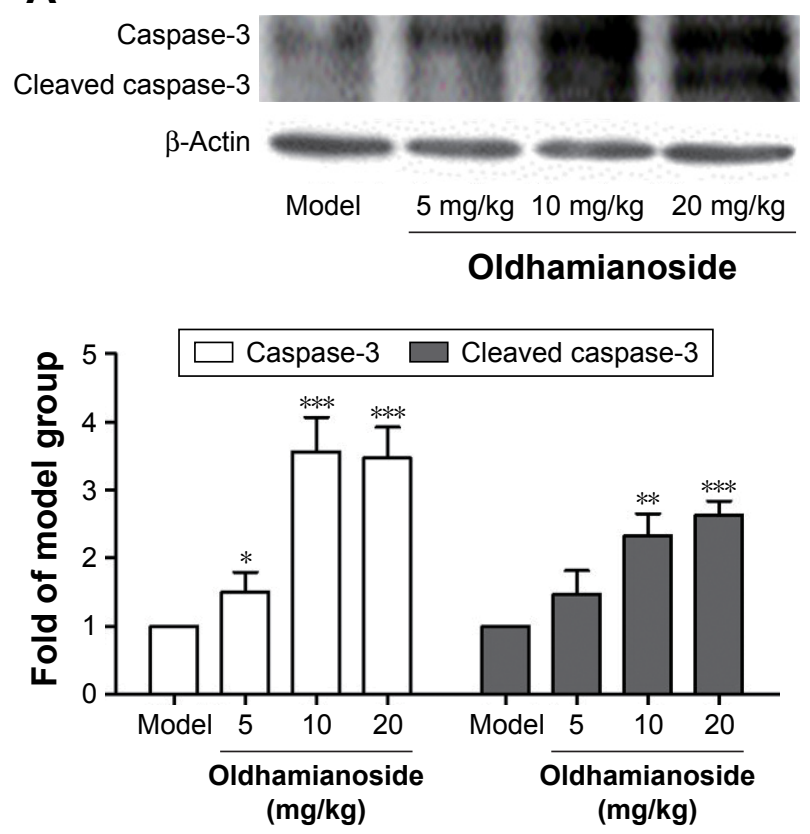

B
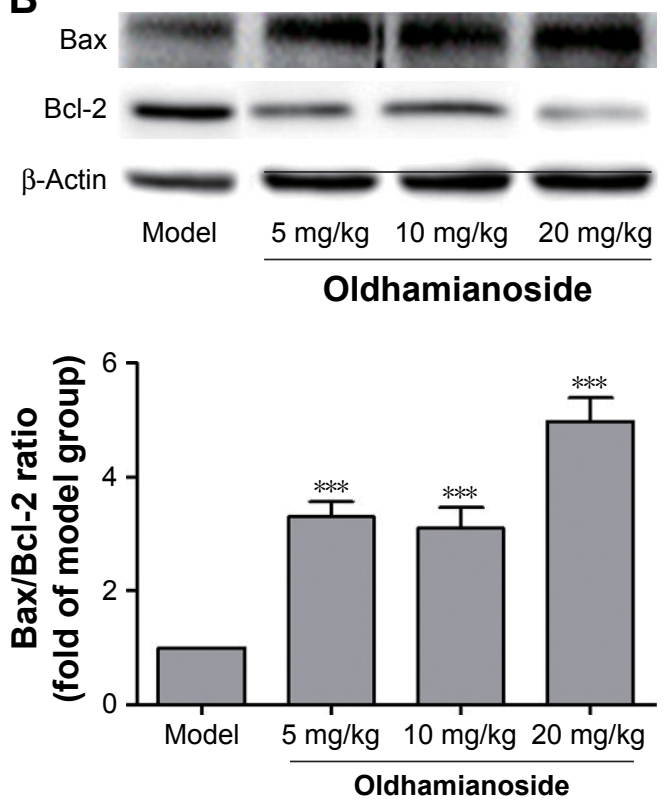

Figure 5 Oldhamianoside modulated apoptosis-related proteins expressions in ovarian tumorigenesis.

Notes: (A) Expression of caspase-3 in tumor tissues from different groups and (B) expression of Bcl-2 and Bax in tumor tissues from different groups. Bars indicate mean \pm SD. Experiments were performed in triplicate separately. $* P<0.05, * * P<0.01$, and $* * * P<0.001$ vs the model group. 
Apoptosis-inducing agents are being investigated as tools for the management of cancer treatment. In mammalian cells, apoptotic cell death is critically governed by the balance between anti-apoptotic proteins, such as Bcl-2 and Bcl-xL, and pro-apoptotic proteins, including Bax and Bak. ${ }^{20,21}$ The induction of apoptosis is usually associated with downregulation of Bcl-2 and upregulation of Bax expression in human cancer cell lines. ${ }^{22}$ Similarly, this study also shows that oldhamianoside treatment downregulates the expression of Bcl-2 and upregulates Bax in human ovarian cancer SKOV3 tumor.

\section{Conclusion}

This study provided evidence in vivo that oldhamianoside inhibits SKOV3 tumor growth, decreases the inflammatory response, prevents angiogenesis in tumor, and induces tumor cell apoptosis, suggesting that oldhamianoside is a potential compound candidate for the treatment of cancer and for the prevention of metastasis.

\section{Acknowledgment}

This research received no specific grant from any funding agency in the public, commercial, or not-for-profit sectors.

\section{Disclosure}

The authors report no conflicts of interest in this work.

\section{References}

1. Ha JH, Ward JD, Radhakrishnan R, Jayaraman M, Song YS, Dhanasekaran DN. Lysophosphatidic acid stimulates epithelial to mesenchymal transition marker Slug/Snail2 in ovarian cancer cells via Goi2, Src, and HIF1 $\alpha$ signaling nexus. Oncotarget. 2016;7(25): 37664-37679.

2. Zhang C, Qiu X. Andrographolide radiosensitizes human ovarian cancer SKOV3 xenografts due to an enhanced apoptosis and autophagy. Tumor Biology. 2015;36(11):8359-8365.

3. Romero I, Bast RC. Minireview: human ovarian cancer: biology, current management, and paths to personalizing therapy. Endocrinology. 2012;153(4):1593-1602.

4. Thibault B, Clement E, Zorza G, et al. F14512, a polyamine-vectorized inhibitor of topoisomerase II, exhibits a marked anti-tumor activity in ovarian cancer. Cancer Lett. 2016;370(1):10-18.

5. Wang FL, Sun JY, Wang Y, et al. Oldhamianoside II, a new triterpenoid saponin, prevents tumor growth via inducing cell apoptosis and inhibiting angiogenesis. Oncol Res. 2013;20(8):369-376.

OncoTargets and Therapy

\section{Publish your work in this journal}

OncoTargets and Therapy is an international, peer-reviewed, open access journal focusing on the pathological basis of all cancers, potential targets for therapy and treatment protocols employed to improve the management of cancer patients. The journal also focuses on the impact of management programs and new therapeutic agents and protocols on
6. Luo JG, Kong LY, Takaya Y, Niwa M. Two new monodesmosidic triterpene saponins from Gypsophila oldhamiana. Chem Pharm Bull. 2006;54(8):1200-1202.

7. Luo JG, Liu J, Kong LY. New pentacyclic triterpenes from Gypsophila oldhamiana and their biological evaluation as glycogen phosphorylase inhibitors. Chem Biodivers. 2008;5(5):751-757.

8. Luo JG, Ma L, Kong LY. New triterpenoid saponins with strong alphaglucosidase inhibitory activity from the roots of Gypsophila oldhamiana. Bioorg Med Chem. 2008;16(6):2912-2920.

9. Bai H, Zhong Y, Xie YY, et al. A major triterpenoid saponin from Gypsophila oldhamiana. Chem Biodivers. 2007;4(5):955-960.

10. Zhang W, Luo JG, Zhang C, Kong LY. Different apoptotic effects of triterpenoid saponin-rich Gypsophila oldhamiana root extract on human hepatoma SMMC-7721 and normal human hepatic L02 cells. Biol Pharm Bull. 2013;36(7):1080-1087.

11. Li L, Zhang J, Weng X, Wen G. Genetic variations in monocyte chemoattractant protein-1 and susceptibility to ovarian cancer. Tumour Biol. 2015;36(1):233-238.

12. Ahn JH, Yang YI, Lee KT, Choi JH, Dieckol CJH. Dieckol, isolated from the edible brown algae Ecklonia cava, induces apoptosis of ovarian cancer cells and inhibits tumor xenograft growth. J Cancer Res Clin Oncol. 2015;141(2):255-268.

13. Pal S, Shankar BS, Sainis KB. Cytokines from the tumor microenvironment modulate sirtinol cytotoxicity in A549 lung carcinoma cells. Cytokine. 2013;64(1):196-207.

14. Li Z, Qiu Y, Personett D, et al. Pomalidomide shows significant therapeutic activity against CNS lymphoma with a major impact on the tumor microenvironment in murine models. PLoS One. 2013;8(8):e71754.

15. Bauerschlag DO, Schem C, Tiwari S, et al. Sunitinib (SU11248) inhibits growth of human ovarian cancer in xenografted mice. Anticancer Res. 2010;30(9):3355-3360.

16. Yamamoto S, Konishi I, Mandai M, et al. Expression of vascular endothelial growth factor (VEGF) in epithelial ovarian neoplasms: correlation with clinicopathology and patient survival, and analysis of serum VEGF levels. Br J Cancer. 1997;76(9):1221-1227.

17. Yates-Binder CC, Rodgers M, Jaynes J, Wells A, Bodnar RJ, Turner T. An IP-10 (CXCL10)-derived peptide inhibits angiogenesis. PLoS One. 2012;7(7):e40812.

18. Tang YJ, Sun ZL, Wu WG, et al. Inhibitor of signal transducer and activator of transcription 3 (STAT3) suppresses ovarian cancer growth, migration and invasion and enhances the effect of cisplatin in vitro. Genet Mol Res. 2015;14(1):2450-2460.

19. Martin L, Schilder R. Novel approaches in advancing the treatment of epithelial ovarian cancer: the role of angiogenesis inhibition. $J$ Clin Oncol. 2007;25(20):2894-2901.

20. Shinagawa A, Yoshida Y, Kurokawa T, et al. The potent peptide antagonist to angiogenesis, $\mathrm{C} 16 \mathrm{Y}$, and cisplatin act synergistically in the down-regulation of the Bcl-2/Bax ratio and the induction of apoptosis in human ovarian cancer cells. Int J Oncol. 2011;39(6):1359-1364.

21. Yang HL, Lin KY, Juan YC, et al. The anti-cancer activity of Antrodia camphorata against human ovarian carcinoma (SKOV-3) cells via modulation of HER-2/neu signaling pathway. J Ethnopharmacol. 2013; 148(1):254-265.

22. Yin PH, Liu X, Qiu YY, et al. Anti-tumor activity and apoptosisregulation mechanisms of bufalin in various cancers: new hope for cancer patients. Asian Pac J Cancer Prev. 2012;13(11):5339-5343.

\section{Dovepress}

patient perspectives such as quality of life, adherence and satisfaction. The manuscript management system is completely online and includes a very quick and fair peer-review system, which is all easy to use. Visit http://www.dovepress.com/testimonials.php to read real quotes from published authors. 